\title{
Civilisations
}

Revue internationale d'anthropologie et de sciences

humaines

66 | 2017

L'alcool rituel et les ethnographes

\section{Des rhums et des hommes. Questions d'alcoolisation sur des terrains de recherche en sciences humaines}

Introduction

Delphine Burguet et Olivia Legrip-Randriambelo

\section{OpenEdition}

Journals

Édition électronique

URL : http://journals.openedition.org/civilisations/4165

DOI : 10.4000/civilisations.4165

ISSN : 2032-0442

Éditeur

Institut de sociologie de l'Université Libre de Bruxelles

Édition imprimée

Date de publication : 31 août 2017

Pagination : $9-21$

ISBN : 978-2-9602017-1-0

ISSN : 0009-8140

Référence électronique

Delphine Burguet et Olivia Legrip-Randriambelo, « Des rhums et des hommes. Questions

d'alcoolisation sur des terrains de recherche en sciences humaines », Civilisations [En ligne], 66 | 2017, mis en ligne le 31 août 2020, consulté le 25 février 2021. URL : http://journals.openedition.org/ civilisations/4165; DOI : https://doi.org/10.4000/civilisations.4165 


\title{
Des rhums et des hommes
}

\section{Questions d'alcoolisation sur des terrains de recherche en sciences humaines}

\author{
Introduction
}

\author{
Delphine BURGUET \& Olivia LEGRIP-RANDRIAMBELO
}

\section{Le boire en anthropologie : enquête de sens}

\section{" L'étude des boissons fermentées mène tout droit dans la religion »}

(Mauss 1926: 43)

Ce dossier thématique s'inscrit à la suite des numéros des revues Terrain (1989) et Socio-anthropologie (2004) consacrés au «boire ». Sans exclusive régionale, les articles rassemblés ici questionnent le cadre social des pratiques alcoolisées et de l'ivresse mais aussi de l'approche méthodologique de l'ethnographe sur son terrain face à l'invitation au boire. Il s'agit ainsi de traiter des boissons et du boire dans leurs fonctions symboliques, rituelles et collectives observées par divers chercheurs en sciences humaines et sociales ayant une expérience de terrain et mobilisant les outils méthodologiques de l'ethnographie. L'alcool est envisagé comme une sociabilité qui se détache du champ de la pathologie (Douglas 1987 ; Fabre-Vassas 1989 ; Fainzang 1995 ; Pourchez 2002).

Les médecines humorales accordaient une place de choix, mais une place tout aussi paradoxale au vin rouge : à la fois boisson et aliment, à la fois mets et médicament. Galien le voyait comme un pharmakon ${ }^{1}$, tant remède que poison. Suivant cet oxymoron, les boissons enivrantes peuvent être perçues, en contexte religieux, comme des « toxiques rituels » (Obadia 2004). S'installe ici une ambiguïté dans leurs représentations entre d'un côté des " poisons sacrés », et de l'autre, un accès aux « ivresses divines » (De Felice 1936). Pour Isabelle Bianquis, cette ambiguïté est comme un pont qui lie plusieurs mondes ; aussi envisage-t-elle l'alcool comme un objet-frontière marquant des séparations, notamment entre les genres, les mondes (humain, divin), les corps (santé, maladie), etc. En somme, l'alcool est à penser comme un agent qui opère la liaison entre ces différentes catégories, un sas qui permet le passage entre l'une et l'autre (2012). Le boire est à la fois moteur de puissance sociale et cause de la perte de contrôle où l'ivresse devient déshumanisante. L'apport de l'anthropologie, à l'instar des alcohol

1 Cf. sur ce point le récent colloque organisé en octobre 2017 au Musée des confluences de Lyon par le Laboratoire d'anthropologie des enjeux contemporains (FRE 2002, CNRS - Lyon 2 - ENS Lyon) sur le thème : «Pharmakon. Mondes empoisonnés, approches anthropologiques ». 
studies, est de mettre en lumière les multiples usages de ces consommations éthyliques.

Dans une grande part des travaux anthropologiques, les auteurs ne conçoivent l'alcool que dans deux acceptions : la sociabilité entre les hommes ou la communication avec les dieux (Bianquis 2012 : 12). Nous souhaitons ici dépasser ce clivage et voir dans la consommation d'alcool un processus plus complexe et englobant. Lionel Obadia insiste sur le fait que « les grandes monographies (...) [ne] mentionnent [qu'] à l'occasion des usages alcooliques » et que l' " on ne confère que rarement à ces derniers un statut central dans l'analyse » (2004). C'est à ce contre-pied que se sont attachés les contributeurs de ce numéro.

Ce dossier ${ }^{2}$, structuré en trois parties, offre une diversité d'approches et d'analyses du lien au boire dans un contexte de recherche qualitative. Regroupés sous le titre «Les buveurs invisibles », les cinq premières contributions traitent à titre principal de l'attachement aux dieux, entités surnaturelles et esprits par les usages du boire alcoolisé afin de montrer la sociabilité des hommes à travers des libations, des offrandes et des consommations ritualisées. On voit dans ces contributions le rôle majeur du monde invisible dans l'interprétation des manières de consommer les alcools. Dans cette première partie, les lecteurs apprécieront des études de cas rattachées à des territoires ou des communautés culturelles et/ou religieuses spécifiques comme la société afrocubaine, les Betsileo de Madagascar, les Kabyé du Togo, les Nanaïs du bassin de l'Amour en Sibérie orientale, les Juifs en Europe et les chamanes du Népal.

La deuxième partie intitulée « Le terrain ivre » regroupe trois contributions qui s'attachent à questionner le rapport au terrain dans un contexte de recherche où les auteurs sont confrontés à l'ivresse : la première montre comment l'ethnographe refuse l'invitation à boire alors que les interlocuteurs sont en situation d'alcoolisation répétée ; la deuxième analyse l'ébriété de l'informateur comme objet d'étude ethnologique ; enfin, la troisième questionne l'enivrement du chercheur sur le terrain. Les études de cas nous emmènent en France dans un Centre d'hébergement et de réinsertion sociale (CHRS), à Madagascar dans la région de l'Imerina et dans une communauté punk à Wuhan en Chine.

La troisième et dernière partie, «L'alcool (en)chanté », propose trois études de cas du boire en musique. Ces contributions travaillent le lien entre le boire et les sons musiqués et/ou chantés. L'approche ethnomusicologique montre les possibles liaisons entre des usages sociaux complémentaires et imbriqués : boire, chanter, jouer, danser... Ainsi, il est question d'interroger les pratiques d'alcoolisation chez les Tai Dam du Laos; parmi les danseurs urbains aymara en Bolivie ; enfin, au sein des troupes de marine de l'armée de terre française entre la France et le Gabon.

\section{Les étiquettes du boire}

«L'homme se nourrit de nutriment [...] mais aussi de signes, de symboles, de rêves et de mythes " (Poulain $1997:$ 126)

Jean-Pierre Castelain (1996) pose les bases méthodologiques et épistémologiques pour ouvrir le champ d'une anthropologie du boire. Pour amener une ouverture à cet objet d'étude, il nous renvoie à l'ethnographie comme démarche qu'il différencie de la sociologie et de l'épidémiologie de l'alcoolisme issue de l'hygiénisme. Pour

2 Ce dossier est le fruit d'un premier travail de réflexion élaboré à l'occasion d'un atelier du Congrès de l'Association française d'ethnologie et d'anthropologie en 2015 à Toulouse et qui portait sur la Démesure. 
appréhender la signification sociale de la consommation d'alcool, il faut se poser ces questions :

« Pourquoi, comment, dans quelles circonstances boit-on, en l'occurrence de l'alcool? Quels sont les effets et les conséquences induits pour les consommateurs tels qu'ils se définissent eux-mêmes ? Quel sens ce comportement a-t-il pour eux ?» (Castelain 1996 : 181).

Sur différents terrains parmi les dockers du Havre, dans l'archipel de Saint-Pierre-etMiquelon et les îles bretonnes, il construit une anthropologie du boire qui traite du rôle du collectif sur la consommation et de ses effets comme une fonction de la ritualisation et de la transgression.

A la suite de ce questionnement, le travail collectif sur le boire anthropologique du numéro « Le boire » de la revue Socio-anthropologie (Obadia 2004) apporte une assise conceptuelle et méthodologique que nous intégrons dans notre propre travail analytique. Le numéro avait pour objectif de rendre compte d'une diversité de perspectives et de méthodes, objectif que nous-mêmes avons décidé de questionner à nouveau.

Depuis, plusieurs chercheurs ont plus précisément analysé la notion de liminarité en considérant le boire et l'ivresse comme objet d'étude. L'anthropologie de l'ivresse rituelle et collective est un champ de recherche qui associe les usages sociaux du boire, la symbolique et ses significations. Il faut considérer l'ivresse rituelle comme une action performative qui favorise la communication entre les entités sacrées et les humains, et entre les humains eux-mêmes. Caroline Magny (2008, 2009) et Céline Geffroy (2013) qui ont étudié les pratiques d'alcoolisation dans les Andes traitent de cette liminarité dans l'ivresse, notamment dans un contexte funéraire. En fonction de l'état d'ivresse, les buveurs estiment leur place soit du côté de la maîtrise de soi, soit du côté de la perte d'identité, où la mémoire est altérée. La perte de contrôle rend ainsi visible un déplacement identitaire, proche d'une conduite « démoniaque ». A ce niveau d'ivresse, de mauvais esprits rôdent et prennent possession des corps enivrés. Du point de vue de la corporéité, des métamorphoses psychiques et physiques sont à l'œuvre dans l'ivresse afin de favoriser la communication avec la nature, la surnature, les défunts et les vivants. La consommation d'alcool et l'ivresse qui en découlent offrent aux buveurs la possibilité d'un dialogue qui, sans cela, ne saurait être possible tant les relations sociales sont hiérarchisées dans les sociétés andines.

Pour Elias, qui a lui aussi questionné les usages sociaux du boire alcoolisé, « l'ivresse est une technique liturgique du corps ", à l'instar de ce qu'on peut observer pour les derviches tourneurs (Elias 2016 : 244) ou les médiums en transe de possession. Elle ouvre un accès au divin par un état de conscience modifiée. L'alcool peut accompagner la liturgie sans pour autant être autorisé ou mentionné dans les textes sacrés. Ainsi, une confrérie musulmane turque invoque un dialogue entre Ali et le prophète qui se clôture avec l'absorption « du jus d'un unique grain de raisin par l'assemblée » qui deviendra ivre (Elias 2016). L'alcool est énigmatique, ses effets sont perceptibles mais leur origine est tue. L'alcool sera le dem : le souffle, l'instant ou le sang (Ibid.).

\section{Anthropologie, mesure de l'alcool et démesure rituelle}

L'absorption de gorgées d'alcool et la libation de liquides relèvent généralement de la description ethnographique, de l'ordinaire, du mode mineur du croire (Piette 2003). En ce sens, elle est souvent exclue de l'analyse. Pourtant, sa part de quotidienneté structure le rituel et mérite d'être observée et pensée de plus près pour produire de la connaissance anthropologique et dégager des réflexions en termes de religiosité, d'enjeux de pouvoir et de méthodologie ethnographique. 
L'alcool est contextualisé en fonction de pratiques sociales liées au sacré (et aux marges) ou à l'extérieur de celui-ci. Si l'alcool est un élément ritualisé, sa quantité consommée n'est pas nécessairement imposée par les esprits ou le(s) dieu(x). De ce constat, les possibilités des pratiques d'alcoolisation sont illimitées. En effet, le consommateur boit à la bouteille, au verre, seul ou avec l'assemblée, en son nom ou au nom des esprits qui le possèdent. La fréquence de consommation d'alcool rythme les étapes du rituel (de la présentation du/des esprit(s) à son/leur départ, en passant par les demandes ou sacrifices). Parfois, comme au Brésil notamment, l'absorption de quantité « spectaculaire » d'alcool par un médium en transe et non ivre est considérée « comme une preuve de la véracité de l'incorporation, et donc de l'existence des esprits » (Rougeon et Santiago 2016). Ainsi, les boissons pourraient être un outil de mesure des compétences magico-religieuses du « buveur», autant dans la transgression de leur consommation que dans la gestion de la sobriété. Dans le discours de certains pratiquants, les compétences sont proportionnelles à la quantité d'alcool ingérée et à la maîtrise de soi. Les officiants du culte expérimentent leurs limites par la démesure de la consommation d'alcool. Ils se trouvent ainsi, dans un jeu de bascule permanent, entre normes (sociale, religieuse, genrée, etc.) et excès, qui crée la maîtrise, ou l'absence de maîtrise, démontrant des capacités extraordinaires ou au contraire renvoyant au danger. La démesure de la consommation d'alcool fait partie intégrante de la mesure rituelle et de ses codes. De fait, malgré l'ambivalence de l'alcool (pathos/ethos), nous privilégions son analyse comme un révélateur ou un opérateur du ciment social (Obadia 2004). Autrement dit, la consommation d'alcool tout autant que sa fabrication, par ailleurs, " articulent une multiplicité de relations sociales, culturelles et économiques » (De Vidas et Losonczy 2016).

Boire en société (rituellement ou non) engage une atténuation de l'acceptation et une négociation du refus afin que le buveur se range aux obligations de consommations (façons et mesure). Les refus ou les interdits sont stéréotypés autant que distincts de la consommation d'alcool elle-même. Ainsi l'on boit trop pour une femme, pour un chrétien ou un musulman, pour l'heure qu'il est, pour ce qu'on a dans l'estomac, etc. Les justifications s'inscrivent dans l'unité de mesure ou dans l'espace : boire un petit coup, un verre, faire la tournée des bars, etc., sans que la mention d'alcool n'apparaisse. Pour reprendre le questionnement de Véronique Nahoum-Grappe, "mais qu'est-ce qu'un 'coup'? Une gorgée ? Un verre ? Un geste ? Tout ce que l'on peut boire en une fois ? Comment le mesurer quantitativement ? L'idée de mesure est néanmoins impliquée dans le 'un' de 'un coup' : une unité peut être comptée jusqu'au 'coup de trop'» (1989: 72). Les mentions d'ivresse et les quantités d'alcool consommées sont mesurées et ethnographiées selon des catégories vernaculaires et ne se réfèrent pas à une échelle éthylique. Nous voyons également apparaître un boire genré à travers les capacités à tenir l'alcool pour une femme, à devoir accepter une invitation à boire pour un homme. La consommation d'alcool impute également un vocabulaire genré dont la graduation est elle aussi à échelle variable : une buveuse passera par le stade " pompette » et un buveur montera à la sensation d'ivresse sans s'arrêter à cet échelon.

\section{Se mettre à table par la carte des boissons}

"Un dernier pour la yourte 》 (Abesdris et al., 2013)

Une carte des boissons nous offre la possibilité d'apprécier la qualité des alcools présentés. Après une réflexion sur les échelles variables de la quantification du boire alcoolisé d'un point de vue anthropologique, il est pertinent de s'interroger sur les saveurs, couleurs, parfums, symboles et représentations de ces boissons enivrantes. 
Se mettre à table, c'est participer à la consommation d'alcool, et en même temps, lorsque les interlocuteurs sont à table, ils se livrent. Dans cette perspective, un grand choix d'alcools parcourt ce volume, des distillés aux fermentés, des mondialisés aux confidentiels. Les bouteilles, les verres, les bols s'emplissent de rhums blancs cubains ou malgaches, de whisky en Chine ou à Madagascar, de vodka en Russie ou en France, de bière de malt, de sorgho ou de millet au Laos, en Bolivie, au Népal, de vins rouges en France et de spiritueux ailleurs encore. Au-delà de la diversité de ces alcools qui attisent les papilles ou soulèvent le cœur, nous en venons à l'idée de les catégoriser à la fois par leur représentation sociale, qu'ils soient artisanaux ou industriels, mais aussi selon le choix opéré par les spécialistes du religieux et selon celui des buveurs anonymes.

Les alcools peuvent être industriels d'une part, artisanaux et domestiques d'autre part, mais encore, autochtones ou allochtones, locaux ou importés. Ils revêtent alors des modes de consommation et des représentations différents (Obadia 2004 ; Fiskejö 2010). Sur le continent américain, l'alcool est ritualisé et « lié aux différentes traditions religieuses » (De Vidas et Losonczy 2016). La distinction ne s'arrête pas là, il faut penser l'alcool dans des contextes rituels mais aussi dans des contextes économiques. Ainsi au Népal, « les alcools sont inscrits dans la production agricole, le système économique et les réseaux sociaux » (Obadia, voir l'article de ce numéro). De fait, il est pertinent d'étudier le boire sans l'exotiser par la consommation d'alcool domestique « traditionnel ». En effet, nombre d'interlocuteurs achèteraient volontiers des alcools industriels prestigieux ou de meilleure qualité s'ils en avaient les moyens financiers. On peut relever le cas des rhums artisanaux frelatés causant de nombreux décès à Madagascar ${ }^{3}$, ou encore de la vodka coupée à l'antigel en Mongolie (Lacaze 2004).

\section{Quotidien, alcool et terrain}

L'approche réflexive au fondement de plusieurs contributions de ce dossier apporte des éléments de compréhension des enjeux de l'alcoolisation et de l'ivresse dans un contexte d'enquête qui nécessite la réalisation d'entretiens, d'observations, d'immersions. Les auteurs tentent ainsi de livrer aux lecteurs leurs vécus à la fois impliqués et distanciés, leurs ressentis, leurs goûts ou leurs dégoûts des alcools.

Dans sa posture sur le terrain, l'ethnographe doit gérer la démesure sociale et/ou rituelle, qu'impliquent les situations excessives du boire. Cet aspect méthodologique s'étend au recueil des données, mais également à leur analyse. Si l'observation est participante, la question posée est celle du refus ou de l'acceptation de s'engager dans la consommation de l'alcool. Comment l'ethnographe gère-t-il l'invitation à boire et éventuellement sa propre ivresse ? L'idée d'intégration doit alors être pensée : l'observateur s'intègre ou est intégré au rituel et/ou au groupe, notamment par la consommation de l'alcool au risque de perdre sa posture d'observateur; ou bien l'observateur évite l'intégration pour limiter la consommation d'alcool, au risque cette fois-ci de perdre la place qui lui a été assignée.

A cela s'ajoute la gestion de la démesure des participants observés. Certains auteurs de ce volume questionnent l'utilisation des données recueillies qui, par l'ivresse des interlocuteurs, semblent incohérentes (Burguet, Amar). L'ethnologue peut identifier et mesurer l'enivrement sans qu'il soit perçu comme tel par les consommateurs et/ ou les observateurs. Les auteurs interrogent alors les définitions de l'ivresse (FabreVassas 1989), et plus largement de la transgression, au regard, par exemple, de codes sociaux variables en lien au boire. Les jugements peuvent alors être autant péjoratifs

3 Selon des données extraites de nos terrains malgaches. 
que mélioratifs. Comment mesurer l'ébriété ? L'alcoolisation telle que nous l'entendons n'est pas nécessairement interprétée comme une déviance par l'analyse ou dans les discours des membres du terrain. De fait, les questions posées ici s'inscrivent dans une réflexion anthropologique du boire.

Dès lors, on se détache du modèle pathologique, comme énoncé dans notre parti pris analytique. Il n'est plus question de démesure imposée par un « contrôle de l'alcool, mais un contrôle par l'alcool » dans le contexte rituel (Elias 2016 : 248). Le lien entre l'ivresse et le rituel est alors à analyser en des termes plus complexes et dans un déplacement de la problématique par rapport à l'ivresse pathologique.

Avec le concept méthodologique et théorique d'intoxication participante (participant intoxication), Magnus Fiskejö nous invite à repenser le boire au regard de la méthode ethnographique (2010). Il introduit une réflexion complexe sur la consommation d'alcool par l'ethnographe. L'expérience du boire par le chercheur en contexte d'observation participante permet la production et le recueil de données grâce au partage collectif, à la commensalité et au vécu émotionnel, rituel ou festif. Comme par une « imprégnation alcoolique » (Jolly 2003 : 108), l'ethnographe s'imbibe de l'expérience sociale de ceux qu'il observe. La construction et l'organisation sociale se jouent autour du verre : une consommation partagée a des vertus ethnographiques d'immersion, mais elle ouvre aussi à des observations centrales pour une analyse des dynamiques sociales. L'intoxication participante est alors un pendant de l'observation participante (Fiskejö 2010 : 111). La consommation d'alcool dans le cadre religieux déplace les codes : « ce ne sont plus ceux qui boivent qui sont frappés de soupçon, mais bien ceux qui ne boivent pas » (Elias 2016 : 250), pour les adeptes, bien sûr, mais pour l'ethnographe également.

\section{Qui sont ces ethnographes qui trinquent?}

«Un petit verre de rhum » (Lévi-Strauss 1984 : 458-459)

Claude Lévi-Strauss (1984 : 458-459) décrivait dans Tristes tropiques ses préférences d'arômes entre différents rhums distillés à la Martinique. Ses prédilections gustatives qui relèvent davantage du journal ethnographique pourraient être celles d'autres chercheurs sur le terrain. Ainsi, certains auteurs de ce numéro n'émettent pas de préférence sur les boissons avec lesquelles ils ont trinqué sur leur terrain, d'autres en revanche consignent certaines expériences du boire alcoolisé. Du fait que les ressentis ne font généralement pas partie des méthodes d'analyse ethnologique, les auteurs évitent d'émettre un avis ou une préférence de goût sur ce qu'ils boivent. Souvent, l'ethnographe va faire le choix de mentionner le goût du sorgho parce que celui-ci est considéré comme particulier, alors que la bière ne sera pas détaillée, car son parfum est supposé connu de tous. Il en est de même des rhums industriels et artisanaux fort différents en taux d'alcool et en parfum. Les premiers sont plutôt vanillés et sucrés, alors que les seconds, appelés communément " tord-boyaux », ont des degrés d'alcool très élevés et râpent la gorge quand ils sont avalés. Pourtant, l'alcool est bu par les ethnologues et leurs interlocuteurs « entre délices et dégouts » (Poulain 1997), selon la temporalité, la nécessité rituelle et sociale, mais aussi suivant les volontés des esprits « comme si ceux-ci avaient leurs goûts et dégoûts propres " (Cobbi 1991 : 114). Dans ce numéro, les auteurs donnent leur avis sur certaines saveurs alcoolisées, car ils ont eux aussi leurs goûts et dégoûts. Ainsi Marie-Pierre Lissoir intègre à sa réflexion méthodologique son aversion pour la bière qui lui est offerte ; Olivia Legrip-Randriambelo réfléchit à la manière dont elle a dû composer avec l'odeur qu'elle dégageait après des libations de rhum artisanal. Ceci ouvre aux questions des (dé)plaisirs du terrain comme expérience sociale, des sensibilités ou encore du rapport au corps des consommateurs, voire à l'absence de 
prise en considération du corps des ethnographes.

Les acteurs du boire mobilisés dans la première partie s'abreuvent tant physiquement que spirituellement. Les cinq contributeurs évoquent des buveurs visibles et invisibles : les officiants de culte, les croyants et les entités religieuses elles-mêmes. Afin d'ouvrir le questionnement à d'autres études de cas que celles recensées dans ce numéro, nous pouvons référencer le travail de Pédron-Colombani au Guatemala sur la matérialisation du boire visible/invisible : les statues de San Simon boivent visiblement l'alcool qui leur est offert par l'orifice buccal percé dans la sculpture. Elles sont équipées d'un dispositif qui sacralise l'alcool bu : le breuvage qui passe de la bouche vers l'arrière de la statue s'écoule pour être récupéré par les officiants du culte (Pédron-Colombani, 2004 : 140-141).

KATERINA KeRESTETZI questionne les rituels du palo monte afro-cubain au cours desquels de nombreuses variétés d'alcool sont consommées, mais aussi versées, crachées, soufflées, enflammées, enduites, toujours selon des codes religieux. S'il est consommé de multiples manières, l'alcool est néanmoins extrêmement codifié dans ses usages. Les médiums boivent à outrance et la pathologie alcoolique qu'ils présentent est attribuée aux morts qui les possèdent. L'alcool est ici avalé par l'officiant mais l'ivresse est celle de l'esprit. Katerina Kerestetzi analyse l'alcool comme un véritable outil rituel. Elle développe cette étude de cas ethnographique sur base de l'anthropologie classique pour ensuite proposer une perspective cognitive originale et pertinente dans ce champ. Ainsi, l'auteure se détache de l'analyse de l'alcool strictement éthylique ou rituel pour le penser comme un objet multifonctionnel aux fortes propriétés matérielles et aux potentialités sociales. Les affordances de l'alcool dépassent la dimension du boire pour rejoindre des sphères plus complexes, comme la mise en scène spectaculaire du rituel (l'alcool est inflammable), la dimension thérapeutique et purificatoire (l'alcool est désinfectant), etc. Ceci va avoir une conséquence sur les actions rituelles, sur les techniques du corps des adeptes du palo monte mais également, hors de la sphère religieuse, sur les fabricants de spiritueux qui vont modifier l'appareil liturgique. L'alcool comme objet rituel mineur devient une porte d'analyse de faits majeurs et collectifs.

Olivia LEgRIP-RANDRIAMBELo présente un aspect des usages du boire inédit en articulant le bien boire et le mal boire dans un contexte religieux du pays betsileo, à Madagascar. Son analyse apporte un éclairage sur les manières de boire lorsque les devins-guérisseurs sont les médiateurs des ancêtres et sur les représentations contradictoires et ambivalentes des signes visibles de l'ivresse. L'auteure confronte deux situations ethnographiques afin d'articuler les regards d'appréciation du boire des patients qui consultent ces guérisseurs possédés afin que ces derniers proposent une solution à leurs maux. Elle offre une lecture des fonctions rituelles de l'usage du rhum dans ce contexte religieux qui convoque les esprits ancestraux, mais son analyse dépasse ce premier niveau de lecture, notamment en décryptant les représentations du boire, qui repose symboliquement sur ces fonctions rituelles fondamentales. Ainsi, elle saisit la possible démesure du boire rituel qui montre des pratiques d'alcoolisation décalées de la symbolique de l'alcool. Elle apporte une analyse sous le prisme du boire genré en présentant les usages du rhum et les façons de boire de Sainte, une jeune guérisseuse qui prend des allures viriles lorsqu'elle est possédée par l'esprit d'un ancêtre royal masculin. Intervient ici l'idée de transgression sociale et genrée dans le boire rituel. A l'inverse, l'auteure nous parle de RaJaona lui aussi thérapeute et désorcelleur mais dont la réputation est abîmée. Dans ce cas, malgré les fonctions symboliques de l'alcool, l'ivresse démesurée du médiateur est mal vue par les patients qui associent l'ivresse visible à une faiblesse du corps performatif.

Après une lecture symbolique de l'ivresse religieuse manifestée par les ancêtres 
malgaches, la démesure de l'alcoolisation se rencontre également chez les Kabyé du Togo qui emplissent les corps d'enfants de bière de sorgho. Boisson cérémonielle qui accompagne tous les évènements individuels et collectifs, elle est étroitement liée aux cycles rituels humains et agricoles dont le fondement religieux est en lien avec le culte des ancêtres de type domestique et lignager. Si, à Madagascar, le devinguérisseur possédé absorbe le rhum pour satisfaire les ancêtres, au Togo, la bière de sorgho emplit le corps des dieux. MARIE DAUGEY propose une lecture minutieuse et approfondie d'un rituel de libation qui consiste à faire boire en grande quantité la boisson à un enfant qui représente le « grand prêtre », préposé au culte aux divinités du territoire. Ce rituel accompagne le développement final du petit mil et l'annonce du commencement prochain des initiations masculines et féminines. L'auteure développe l'aspect symbolique de cette cérémonie et les aspects formels du déroulement de la sur-consommation d'alcool par l'enfant-prêtre. Son analyse permet une relecture de la représentation de l'enfant et de sa corporéité ainsi que de la symbolique des matières comme récipient, contenant, réservoir des dieux.

Dans son article, ANNE DALles propose de coupler l'approche ethno-historique à la perspective ethnologique. Elle suit le fil de la consommation d'alcool rituel, ici de la vodka, jusqu'au changement de mode de consommation, lorsque l'absorption de vodka sort du contexte religieux. Les Nanaïs du bassin de l'Amour en Sibérie orientale ont cessé d'adjoindre la vodka aux rituels chamaniques. Le religieux et, par répercussion, l'alcoolisation se sont russisés. L'auteure ethnographie l'alcool dans sa dimension pathologique et dans la sphère du privé. Néanmoins, ce texte s'inscrit dans une réflexion sur l'alcool rituel par le lien discursif que maintiennent les Nanaïs avec le recours obligé à la vodka dans les anciens rituels chamaniques. Le déplacement symbolique de l'alcool se donne à voir en termes d'opposition : de la sphère publique à la sphère privée, du religieux au pathologique, de la fête à la dépression, des chamanes aux conduites suicidaires. Dès lors, cet article permet de retracer les modifications et les disparitions de pratiques rituelles. Là encore, les lecteurs pourront saisir l'importance de voir dans la consommation d'alcool un geste et un objet qui sont autrement plus importants socialement qu'une addiction pathologique et/ou un objet rituel. Anne Dalles nous entraine dans les liens des Nanaïs à l'alcool avec une profondeur historique qui fait écho aux pratiques contemporaines malgré la disparition des chamanes. La consommation d'alcool reste associée au chamanisme par les mouvements religieux évangéliques charismatiques qui les combattent et y voient des marqueurs des maux de la société nanaï.

Enfin, l'article de LiONEL OBADIA interroge les fonctions de l'ivresse en contexte religieux et questionne le boire ritualisé selon des matières et des manières de faire. Pour cela, l'auteur inscrit son positionnement théorique selon une perspective comparative qui exploite des données ethnographiques localisées et circonscrites (le Pessah juif en Europe et le guru-puja chamanique au Népal) qui présentent des contrastes morphologiques, fonctionnels et symboliques dans les modalités de l'alcoolisation. A partir de ces deux terrains fort éloignés, l'auteur montre que l'alcoolisation assure des fonctions différentes sous la surface de similitudes formelles. Autrement dit, si l'ivresse éthylique se donne à voir de manière semblable dans les deux cas, les modulations d'intensité et de sens changent d'un rituel à un autre. Son argumentaire pour une approche comparative trouve sa pertinence ici, dans le dépassement de la simple lecture des manières de boire. Ainsi, il saisit le sens du boire rituel alors que l'alcoolisation ritualisée est un trait commun à ces deux terrains. Pour le rituel du Pessah qui s'inscrit dans un monde sémitique et monothéiste, l'alcoolisation est codifiée par les textes sacrés et associée à une ivresse réalisée pour et par le rite. Le 
caractère obligatoire du boire durant le rite pour tous les participants, dont les enfants qui ont atteint leur maturité spirituelle, suppose une ivresse collective qui participe à une sociabilité émotionnelle. Ce rituel, comme beaucoup d'autres dans le judaïsme, comprend des modalités d'alcoolisation normées, codifiées et centrales et qui semblent prendre leur charge symbolique héritée des Hébreux, civilisation de la vigne dans un environnement agricole. A l'inverse, dans le guru-puja, issu de la société himalayenne, bouddhiste et chamanique, l'alcoolisation apparaît comme une pratique qui facilite le déroulement mais elle reste marginale. L'usage de l'alcool dans ce contexte a une fonction principalement récréative pour le chamane et les participants au rite, lors de temps « faibles ». Si l'on boit beaucoup, c'est surtout pour la convivialité festive liée à l'activité rituelle, aux interstices de séquences rituelles d'importance.

Avec une soif de connaissances, l'ethnographe accepte toujours de boire les paroles de ses interlocuteurs, mais l'invitation à partager un verre d'alcool, substance qui justement délie les langues, ne va pas de soi. C'est à la question des métaphores du boire, à cette nuance entre l'absorption des mots et des liquides, ainsi qu'à la justesse de l'acceptation ou du refus de boire que s'attachent les auteurs de la deuxième partie, «Le terrain ivre ». Accepter ou refuser le verre d'alcool que l'on vous tend, c'est adopter une posture, et s'engager d'une certaine façon sur le terrain ; ce n'est pas simplement boire, mais s'initier et plonger dans de nouvelles modalités d'interaction.

Le premier article est en ce sens original car il montre comment le chercheur refuse l'invitation à boire ; un refus construit selon une méthodologie adaptée au contexte. FRANCIS VERNEDE amène ainsi le lecteur sur son lieu d'enquête où se mêlent invitation, questionnement, adaptation, refus et réajustement. C'est dans un Centre d'hébergement et de réinsertion sociale (CHRS) qu'il analyse sa place de chercheur dans les conduites d'alcoolisation ritualisées des usagers du centre. La plupart alcooliques, ces usagers l'invitent à consommer alcool et autres substances durant des temps d'apéritif, dont les pratiques socialisantes et ritualisées amènent à questionner la posture de l'observateur face à l'ivresse et au caractère illicite des consommations. Pour lui, refuser de boire de l'alcool avec les usagers du centre, c'est garder la bonne distance, garder une place idéale. L'article met en avant une méthodologie d'enquête, une façon d'agir et d'interagir dans un contexte ethnographique spécifique. Outre l'ethnographie minutieuse d'un apéritif comme espace et temps ritualisés, l'auteur livre sans gêne la façon dont il a bâti son rapport au terrain, en fonction des attentes des travailleurs sociaux et des usagers. Par son bricolage ethnographique, le chercheur ajuste ses conduites sur le terrain en fonction de ses principes et de son éthique scientifique, mais aussi en fonction des sollicitations des interactants. L'enjeu est de taille car la décision de boire ou de ne pas boire oriente la suite de la recherche, et peut modifier la qualité et la quantité des données produites. Il montre que sa place, celle qu'il a décidé de s'attribuer, n'est alors pas idéale mais idéalisée : refuser de boire de l'alcool avec les usagers lors d'apéritifs le situe en dehors. Il questionne de fait les notions de dilemme et de tension que subit parfois l'ethnographe lorsque le terrain se compose de pôles sociaux opposés. La réflexivité de l'auteur apporte également des outils de compréhension sur les pratiques du boire dans le CHRS, car l'ethnographie de la ritualisation du boire met en exergue une hiérarchisation des groupes qui composent le centre (professionnels/usagers) et une asymétrie dans les pratiques informelles qui amènent l'ivresse.

L'article de DelPhine BuRguet part du postulat classique développé dans d'autres ethnographies de ce volume : le guérisseur possédé par un esprit qui boit ne ressent pas l'ivresse, c'est l'esprit qui possède qui reçoit les effets. Ce constat étant fait, Delphine Burguet propose de déplacer le regard ethnographique pour le porter de l'autre côté des matériels technologiques permettant le recueil des données. Le contexte religieux 
associé à celui de la consommation mutuelle d'alcool incite l'ethnographe à recourir aux dispositifs photographique et filmique. Le second regard sur ses données, différé cette fois, a imposé à l'auteure une nouvelle réflexion sur l'alcoolisation de son interlocuteur. Si au cœur du rituel, l'alcoolisation du devin-guérisseur est celle de l'esprit qui possède - la théorie l'emporte sur l'empirique -, avec le retour différé sur les images du rituel, l'alcoolisation du devin-guérisseur semble plus concrète - l'empirique l'emporte sur la théorie. Dès lors, le corps de l'officiant du culte parle pour lui et l'analyse filmique met en exergue une gestuelle titubante et un visage marqué. Entre analyse des matériaux filmiques et restitutions aux lecteurs par le biais de captures d'écran, il nous est donné de saisir la manière dont l'ethnographe a structuré sa réflexion et la façon dont un chercheur opère le tri et le retour sur ses données de terrain. Cette idée, somme toute évidente concernant l'enquête ethnologique, n'est pas toujours révélée ni assumée par les ethnologues dans leurs écrits.

NATHANEL Amar aborde quant à lui l'alcoolisation d'une communauté punk à Wuhan en Chine et la place de l'ethnographe vis-à-vis de son objet d'étude. Contrairement à la posture distanciée de Francis Vernède quant à l'invitation à boire de l'alcool, Nathanel Amar accepte l'invitation de ses interlocuteurs punks et embrasse dans le même temps un certain nombre des codes culturels et artistiques qui les caractérisent, comme le tatouage. A travers une ethnographie serrée, l'article aborde tout d'abord la question des usages de l'alcool chez les punks, activité ritualisée et associée à des pratiques spécifiques de groupes dits marginaux. Ils sont les acteurs d'une contre-culture musicale mais surtout d'une contre-société avec ses codes, lieux de sociabilité et rituels. L'auteur montre comment, à travers la dimension festive du punk et en particulier la consommation d'alcool, se manifestent les spécificités du jianghu, composé de toutes les figures ambivalentes d'un contre-pouvoir. Dans cette communauté punk, l'alcool joue le rôle d'objet d'échange dans des rapports de don et de contre-don, tout comme les cigarettes et la marijuana. Par la suite, l'auteur propose une approche réflexive de son expérience du terrain en lien avec le boire alcoolisé et dévoile sa longue immersion ethnographique. Composante essentielle de l'activité punk, l'invitation à boire n'a pas pu être refusée par le chercheur, au risque de ne pas pouvoir relire ses notes de terrain écrites en état d'ivresse ! Mais l'auteur explique qu'il a gagné sa place d'insider qui résiste à l'alcool afin de mener jusqu'au bout, corps et âme, cette étude sur les punks chinois.

L'alcool, dans sa dimension rituelle, apparaît et est bu en marge ou au cœur de pratiques festives. Dans la troisième et dernière partie, l'alcool récréatif ou partie prenante du rituel accompagne le chant : trois ethnographies nous invitent à penser l'alcool par le biais de l'ethnomusicologie. A priori, cette branche de l'anthropologie et l'objet alcool semblent fort éloignés. Les trois auteures nous démontrent cependant combien il est pertinent de croiser la pratique du chant et la consommation d'alcool, car l'une institue l'absorption de l'autre et la seconde organise ou plutôt rythme la première.

Marie-PierRe LissoIr nous emmène au Laos, chez les Tai Dam du nord, dans les provinces de Bolikhamxay et de Houaphan. L'auteure présente tout d'abord les rôles et significations du boire en rapport avec la pratique du chant et les relations sociales qui s'y jouent, notamment les usages genrés et statutaires. L'alcool de riz distillé (laolao) et de la bière de riz fermentée en jarre (laohay) coulent à flot dans les moments festifs populaires et religieux. L'auteure montre que le chant et l'alcool, tout comme l'abondance des mets, sont des éléments essentiels à la réussite d'une fête. Et il est bon de montrer son ivresse pour témoigner de la joie de participer. Ainsi, son analyse questionne ces usages festifs et rituels comme des éléments fondamentaux du renforcement du lien social familial et collectif. Alors que Nathanel Amar ne peut 
exploiter ses notes de terrain illisibles, écrites en état d'ivresse, Marie-Pierre Lissoir qui prend des notes durant une cérémonie, se fait remarquer par le fait qu'elle n'est pas assez ivre (elle arrive encore à écrire) : ainsi, elle ne satisfait pas suffisamment ses hôtes. Dans ce rapport d'altérité fondé sur les signes de l'ivresse et qui montre des formes de pression sociale dans les manières de boire, il n'est plus question de se démarquer ou de se distancier par l'intermédiaire d'une posture ethnographique, mais de se confondre aux autres par l'ivresse collective. L'auteure propose aussi une lecture réflexive de ses conduites vis-à-vis de l'invitation à boire. Perçue tout d'abord comme une contrainte, l'invitation à laquelle l'ethnographe répond positivement donne lieu à de nouveaux liens sociaux et enjeux de socialisation pour elle et ses interlocuteurs. Ses consommations d'alcool successives et ses états visibles d'ivresse sur ce terrain long et dense ont amené Marie-Pierre Lissoir à des changements progressifs de statuts. Allant jusqu'à servir ses hôtes en alcool, une pratique qui marque un statut privilégié pour celui qui sert à boire, l'auteure s'adonne aux airs chantés et aux effluves alcoolisées.

Nos pérégrinations autour de l'alcool nous amènent ensuite dans les Andes, où LAURA FLÉTY nous invite à circuler entre les caisses de bières au cours de festivités. La dimension rituelle de la consommation et de la circulation d'alcool au sein d'un groupe de danseurs urbains aymara, en Bolivie, s'entend dans ce contexte ethnographique à la suite des nombreuses et fameuses études sur les notions de don et de contre-don. L'auteure poursuit ces réflexions en resituant les faits sociaux construits autour des dons de caisses de bières, mais aussi bien entendu de leur réception. Comment ces caisses de boissons révèlent une symbolique d'ancrage à un groupe social ? Comment ces dons alcoolisés peuvent-ils être vecteurs de conflits ou de résolutions de conflits ? Comme dans le cas du palo monte cubain, il sera ici question de la mise en scène spectaculaire d'un rituel organisé et structuré autour de l'objet alcool. Seront traitées la performativité rituelle (offrande à une divinité) et son extension au social (organisation de la fête qui se déroulera l'année suivante). L'alcool est à analyser dans le rapport à l'espace : les caisses de bière circulent, mais les participants à la fête circulent eux aussi autour des caisses matérialisant des constructions éphémères et des manières de boire. L'alcool s'immisce dans des dispositifs spatiaux mais aussi corporels, par la transformation impliquée par les pratiques dévotionnelles. Enfin, les transactions d'alcool constituent les liens du groupe de danseurs : les transferts de bière créent le groupe ; les donateurs d'alcool s'inscrivent dans une relation de durée au sein du groupe tant du point de vue économique que rituel.

Adeline Poussin nous propose une immersion sur un terrain peu ouvert et discret au regard des ethnologues : le monde social des armées et plus précisément le temps de commensalité arrosé d'alcool vinifié des «p'tit déj” colo » hautement ritualisés et où le vin rouge et sa représentation virilisée prennent une place centrale. L'auteure a ethnographié les temps conviviaux de ces repas entre la France et le Gabon, suivant les troupes de marine de l'armée de terre française. Les «p'tits déj' colo » mêlent la consommation d'alcool à des chants hérités de l'ancienne appellation de ces bataillons de « Troupes coloniales ». L'âpreté du vin rouge est associée aux oignons crus et aux sardines lors de ce repas matinal, ces trois ingrédients constituant la part ritualisée de l'ingestion. S'y ajoutent des viennoiseries, de la charcuterie et des boissons non alcoolisées et dépourvues de sens pour les Marsouins. Cet évènement crée la cohésion du groupe et impose l'absorption d'au moins un verre de vin lors du rituel d'ouverture appelé « la poussière ». Ce rituel répond à des codes stricts de posture collective, dont la prise du verre et son port aux lèvres font partie. S'ensuit une consommation plus libre et des chants. La consommation d'alcool proscrite pendant le service est possible par son aspect rituel dans ces temps de commensalité qui relèvent pourtant du temps 
de service. Le temps rituel autorise le contournement et apparaît dans les paroles des chants analysés par Adeline Poussin. Les corps des marins sont alors à l'épreuve de l'alcool mais les militaires doivent résister à l'enivrement. L'auteure nous démontre comment la consommation d'alcool est tantôt régulée tantôt encouragée par les Troupes de Marine, en situant le vin rouge dans l'histoire de ces troupes et en le constituant ainsi comme leur représentation : passé maritime, endurance physique, virilité.

L'idée du bateau ivre sur les flots ou à quai est repensée par Adeline Poussin en clôture de ce volume.

\section{Conclusion. A lire sans modération}

Objet d'étude anthropologique, le boire alcoolisé apporte un éclairage sur les usages culturels, religieux et ritualisés de la consommation d'alcool. Selon une approche spécifique des sciences humaines et sociales, le caractère pathologique des alcools considérés comme produit toxique, notamment au regard de la biomédecine, est éloigné. Sans rejeter la dangerosité de la consommation d'alcool, ce numéro s'attache à construire une réflexion des usages sociaux de ces boissons et de ceux et celles qui les offrent, les aspergent, les boivent. L'idée sous-jacente aux études de cas livrées ici est celle de la liminarité dans les représentations que l'on porte sur les alcools : à la fois toxique-médicament, socialisant-excluant, festif-nocif, divin-démoniaque, puissancefaiblesse... On se rend compte que les contours de cet objet-frontière sont mouvants, fluctuants en fonction des consommateurs, des spectateurs, des observateurs. Dans ce jeu d'équilibre ou de bascule, la socialisation est la notion centrale discutée dans ce numéro où les liquides font office de liant. On le voit également dans les contributions qui jettent un regard distancié sur les terrains alcoolisés : ne pas boire ce liant ne délie pas les langues mais au contraire délie les relations entre informateurs et ethnographes. Tout comme les participants au moment ritualisé, qu'il soit un apéritif, un p'tit déj', un rituel de guérison, une cérémonie religieuse, un concert de musique punk, l'ethnographe est invité à boire comme ceux qu'il observe. L'invitation est alors plus ou moins prononcée, répétée ou presque forcée afin de donner à l'observateur un nouveau statut social intégrateur. Nous l'avons vu, l'enjeu pour les consommateurs et les abstinents est un enjeu d'intégration sociale : on boit pour communier, prier, danser, parler, communiquer, s'identifier, s'intégrer, ethnographier. Tout comme d'autres substances toxiques, les alcools portent des fonctions sociales parfois fondatrices des codes rituels d'une société ou d'une communauté. Alors que les contributions de Delphine Burguet, d'Anne Dalles, de Marie Daugey, de Laura Fléty, d'Olivia Legrip-Randriambelo, de Katerina Kerestetzi et de Lionel Obadia montrent le lien étroit avec le domaine du religieux, les études de Nathanel Amar, de Marie-Pierre Lissoir, d'Adeline Poussin et de Francis Vernède s'en éloignent et insistent davantage sur les notions identitaires et communautaires entre pouvoir et contre-pouvoir.

Il s'entend que ce dossier n'a pas pour objectif de couvrir toutes les problématiques anthropologiques liées au boire. Il s'attache à approfondir les réflexions à partir des thématiques qui ont été privilégiées. Nous avons préféré une cohérence du point de vue du traitement de l'objet d'étude et une diversification concernant les terrains étudiés. Ainsi, les contributeurs questionnent le boire avec finesse et non pas dans son acception imprécise englobant l'alcool comme le lien social par excellence, comme un élément pathogène ou comme un simple attribut religieux. Ils ambitionnent d'apporter aux lecteurs des clés d'analyse et des pistes théoriques pour creuser la question de l'ivresse, de l'alcoolisation et du boire sur des terrains ethnographiques. Nous espérons pouvoir nourrir les questionnements en cours et offrir des éléments à l'anthropologie du boire. 


\section{Références citées}

BiAnQuis, Isabelle, 2012. L'alcool. Anthropologie d'un objet-frontière. Paris : L'Harmattan.

CoBBI, Jane, 1991. « Dieux buveurs et ancêtres gourmands », L'Homme, 31 (118), pp. 111-123.

Castelain, Jean-Pierre, 1996. « La quête des mots. De quelques usages de l'alcool dans la France de l'Ouest », Communications, 62, pp. 181-193.

De Felice, Philippe, 1936. Poisons sacrés, ivresses divines. Essai sur quelques formes inférieures de la mystique. Paris : Impr. Louis Bellemand et fils.

De VidAs, Anath Ariel et Anne-Marie Losonczy, 2016. « Alcool et ivresse en Amérique - Approches historiques et anthropologiques », Nuevo Mundo Mundos Nuevos, Débats, mis en ligne le 25 janvier 2016, <http://journals.openedition.org/nuevomundo/69032>

Douglas, Mary, 1987. Consuctive Drinking. Perspectives on Drink from Anthropology. Cambridge : Cambridge University Press et Paris : Maison des sciences de l'homme.

Elias, Nicolas, 2016. « La discipline de l'ivresse dans une confrérie musulmane de Turquie », Archives de sciences sociales des religions, 174 (2), pp. 241-254.

FABre-VAsSas, Claudine, 1989. « La boisson des ethnologues », Terrain, 13, pp. 5-14.

FAinZAng, Sylvie, 1995. «L'alcool, les nerfs, le cerveau et le sang », L'Homme, 135 (3), pp. 120-125.

FiskesJö, Magnus, 2010. «Participant Intoxication and Self-Other Dynamics in the Wa context », The Asia Pacific Journal of Anthropology, 11 (2), pp. 111-127.

GefFroy, Céline, 2013. Boire avec les morts et la Pachamama. Une anthropologie de l'ivresse rituelle et festive dans les Andes boliviennes. Thèse de doctorat, Université de Nice Sophia Antipolis, 601p.

JOLLY, Éric, 2003. « L'ivresse du terrain. Une enquête sur la bière de mil dans la société Dogon (Mali) », in Lionel OBADia (éd.), L'ethnographie comme dialogue. Immersion et interaction dans l'enquête de terrain, pp. 91-136. Paris : Publisud.

Abesdris Julie, Claire Durot, Guillaume Fauvel, Pierre Guibe, Dorothée Lecallier, Philippe Michaud, Arnaud Plat, Olivier Delclos, Dicken Higgins, Brigitte Crost, Munkhzaya Myagmarjav, Tuvshinjargal ToIvgoo, Baigali Tumur-OchiR et Gaëlle LACAze, 2013. « Un dernier pour la yourte. Expériences de Médecins du Monde en Mongolie », Alcoologie et addictologie, 35 (1), pp. 43-50.

LACAZE, Gaëlle, 2004. « Convivialité, consommation d'alcool et catégories de personne chez les Mongols et les Kazakhs », Les Annales de la Fondation Fyssen, 19, pp. 30-47.

LÉVI-STRauss, Claude, 1984 [1955]. Tristes tropiques. Paris : Plon.

Magny, Caroline, 2008. Boire à Churcampa (Pérou) de la production des boissons au sens de l'ivresse. Thèse de doctorat en ethnologie, Université de Nanterre.

MaGnY, Caroline, 2009. «Quand on ne peut plus boire d'alcool ni mâcher de feuilles de coca. Le cas des convertis " protestants » dans les Andes centrales péruviennes », Anthropology of food, S4 | May 2008, mis en ligne le 30 mai 2008, <http://journals.openedition.org/aof/2972>

Mauss, Marcel, 1967 [1926]. Manuel d'ethnographie. Paris : Editions sociales.

NAhoum-Grappe, Véronique, 1989. « Boire un coup... », Terrain, 13, pp. 72-80.

OBADIA, Lionel, 2004. «Le 'boire' », Socio-anthropologie, 15, mis en ligne le 15 juillet 2006, <http:// journals.openedition.org/socio-anthropologie/421>

PÉdron-Clomobani, Sylvie, 2004. Le culte de Maximon au Guatemala. Londres : Periplus Publishing.

PiETTE Albert, 2003. Le fait religieux. Une théorie de la religion ordinaire. Paris : Editions Economica.

PoulaIN, Jean-Pierre, 1997. « La nourriture de l'autre : entre délices et dégoûts », Internationale de l'imaginaire, 7, pp. 115-140.

Pourchez, Laurence, 2002. «L'alcoolisme au féminin à l'île de la Réunion », Ethnologie française, 32 (4), pp. 689-697.

Santiago, Jorge P. et Marina Rougeon, 2017. Manger avec les esprits. Offrandes, partages et soins dans l'umbanda au Brésil et au Portugal. Paris : Editions des archives contemporaines. 